\title{
Influence of Membrane Stress on Seafloor Spreading Driven by Tide-generating Force
}

\author{
Long Chen*,1,2,3, Jianguo Chen, ${ }^{*, 4}$, Qinghai $\mathrm{Xu}^{2,5}$, Wei Guo ${ }^{3,6}$, Dong Ma ${ }^{4}$, Guoling Zhang ${ }^{4}$, \\ Jinsong Liu $^{2,5}$, Shuang Liang ${ }^{3,6} \&$ Lixue $\mathrm{Wei}^{3,6}$ \\ ${ }^{1}$ Hebei Normal University, Shijiazhuang, China \\ ${ }^{2}$ Hebei Key Laboratory of Environmental Change and Ecological Construction, Shijiazhuang, China \\ ${ }^{3}$ Hebei Key Laboratory of Computational Mathematics and Application, Shijiazhuang, China \\ ${ }^{4}$ Research Centre for Analysis and Prediction, Earthquake Administration of Hebei Province, Shijiazhuang, China \\ ${ }^{5}$ College of Resources and Environmental Sciences, Hebei Normal University, Shijiazhuang, China \\ ${ }^{6}$ College of Mathematics and Information Science, Hebei Normal University, Shijiazhuang, China \\ * The two authors have contributed equally to the work. \\ Correspondence: Jianguo Chen, Research Centre for Analysis and Prediction, Earthquake Administration of \\ Hebei Province, Shijiazhuang, China. Tel: 86-186-0321-6806. E-mail: zhuanjiatuijian@126.com
}

Received: July 10, 2012 Accepted: July 23, 2012 Online Published: July 31, 2012

doi:10.5539/esr.v1n2p315 URL: http://dx.doi.org/10.5539/esr.v1n2p315

\begin{abstract}
Although Alfred Lothar Wegener proposed his continental drift hypothesis more than 100 years ago, there has been constant contention regarding the driving source of plate motions. The current geodynamic theory is mainly based on traditional mechanics. The crust is extremely thin in comparison to the earth's radius, which may be caused by a more complex mechanical system: the mechanics of shells. The mechanics of shells reveals the mechanical effect of the tide-generating force on the earth's crust. We present a formula that can be used to calculate the membrane stress resulting from the fluctuation of the mantle tide wave in the crust and estimate its magnitude. Although the tidal force is small, the membrane stress is high. Our investigation suggests that the membrane stress is the fundamental reason for rifting and rupture processes at mid-ocean ridges. It is thus the dynamic source for seafloor spreading and plate motion, which offers a reasonable interpretation of various significant problems that are unexplained by mantle convection theory. And there must be the plate tectonics affected by the tidal force is in the terrestrial planets or other celestial bodies.
\end{abstract}

Keywords: tide-generating force, membrane stress, plate motion, dynamic source, terrestrial planets

\section{Introduction}

Since Alfred Lothar Wegener proposed the continental drift hypothesis (Evans, 1923; Hallam, 1975; Jordan, 1974; Meinesz, 1947; Minster, Jordan, Molnar, \& Haines, 1974; Mobus, 1982; Ranalli, 2000; Scoppola, Boccaletti, Bevis, Carminati, \& Doglioni, 2006), there has been disagreement regarding the formation mechanism, evolution law and the driving forces behind plate motions. No current hypothesis regarding driving mechanisms is proven or convincing (Bird, 1998; Conrad \& Lithgow-Bertelloni, 2004; Forsyth \& Uyeda, 1975; Lithgowbertelloni \& Richards, 1995; Person \& Garven, 1994; Plattner, Malservisi, \& Govers, 2009; Royden, 1993; Vandecar, James, \& Assumpcao, 1995; Wahr et al., 2009).

The present geodynamic theory is mainly based on traditional mechanics. However, the relatively thin crust in comparison to the earth's radius may have more complex source mechanism, and classical theory might be inapplicable here. Instead, the mechanics of shells and the effect of membrane stress(Belytschko, Stolarski, Liu, Carpenter, \& Ong, 1985; Bitbol, Peliti, \& Fournier, 2011; Capovilla \& Guven, 2002; Springer et al., 2010) on the lithosphere system may cause a new revolution in geodynamics.

\section{Data and Methods}

After extensive investigation on the relation between $420747 \mathrm{Ms} \geq 4$ global earthquakes and solid earth tides, earthquakes are concentrated around the $0^{\circ}, 90^{\circ}, 180^{\circ}$ and $270^{\circ}$ phases of the theoretical semidiurnal solid tide. 
Moreover we propose a new method for the short-term forecasting of earthquakes (Chen, Chen, \& Xu, 2012) .

It's found that $M \mathrm{~s} \geq 7$ global earthquakes occur surrounding four earthquake-prone phases of the semidiurnal solid tide(Chen et al., 2012), providing strong evidence that the lunar-solar tidal force is an important factor for trigging earthquakes. As the origin times of earthquakes are controlled by the semidiurnal earth tides, and earthquakes themselves are caused by crustal fractures and plate motions, there must be an inherent relation between the tide-generating force and plate motions, which may provide insight into the driving forces of plate motions.

To describe this relation, we used principles of thin-shell mechanics to analyze the dynamics and influence of the tidal force on the crust. If there were no Moon or Sun, the earth would be stable due to gravity. However, the tidal force produces additional stress within the earth (Figure 1A) (i.e., pressure Pm as mentioned in the continuous incompressible elastic earth model proposed by Kelvin).

A

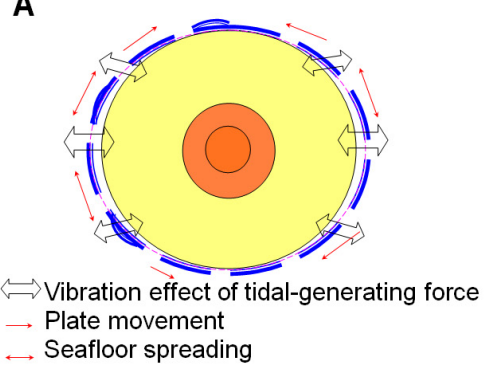

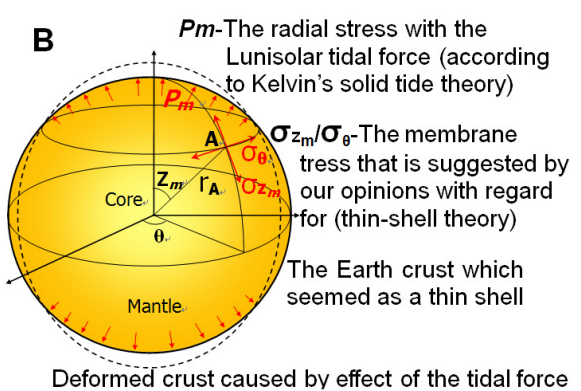

Deformed crust caused by effect of the tidal force

Figure 1. Driving forces of plate motions. (A) The lunisolar tidal force is an important factor for trigging earthquakes, which are caused by crustal fractures and plate motions, indicating that there might be an inherent relation between the tide-generating force and plate motions. (B) We propose that the radial stress causes the membrane stress in the crust through the tide-generating force within the earth

As the crust is extremely thin in comparison to the radius of the earth, the crust can be considered a thin shell and the theory of non-bending moment membrane stress (Belytschko et al., 1985; Bitbol et al., 2011; Capovilla \& Guven, 2002; Springer et al., 2010) can be used to analyze the mechanical effect of the tidal force. Under the tidal force, the upward pressure Pm exerted by the viscoelastic mantle on the crust generates a horizontal distending membrane stress within the crust (Figure 1B), similar to wind causing tension of a boat's sail. Although the tidal force is small, the membrane stress may be large.

Therefore, we combined Kelvin's elastic earth model with thin-shell mechanics to establish a model of the tidal force on the crust and derive a relevant formula.

If there were no Moon or Sun, the earth should be statically balanced. There would exist only a compressive stress in the crust and earthquakes would not occur. However, the lunar and solar tidal forces cause additional stress within the elastic earth. In the theory of Kelvin's earth model, the earth is considered to be a continuous incompressible elastic ball and the tidal stress on any Point A within it by tidal force should be

$$
P=\sum \rho V_{n}(A)+\sum P_{n}(A)
$$

where

where

$$
\begin{gathered}
\sum \rho V_{n}(A)=\sum \rho T_{n}(A)+\rho T^{\prime}(A) \\
\sum P_{n}(A)=-\sum \frac{\rho\left(2 n^{2}+5 n+3\right) \mu}{\left(2 n^{2}+4 n+3\right) \mu+n \rho g R} T_{n}(A)
\end{gathered}
$$

$T_{n}(A)$ is the potential function of tidal force on Point $\mathrm{A}$ within the earth and $T^{\prime}(A)$ is the additional gravitational potential resultant from the earth's deformation. 


$$
T^{\prime}(A)=\sum \frac{3 g}{2 n+1}\left(\frac{r(A)}{R}\right)^{n} \varepsilon_{n} S_{n}(A)
$$

In this formula, $\varepsilon_{n} S_{n}(A)$ is the radial displacement of Point A, which can be calculated using

$$
\varepsilon_{n} S_{n}(A)=\frac{n(2 n+1) \rho R}{2\left[\left(2 n^{2}+4 n+3\right) \mu+n \rho g R\right](n-1)} T_{n}(A)
$$

Therefore:

$$
\begin{gathered}
\rho T^{\prime}(A)=\sum \frac{3 n \rho^{2} R g}{2\left[\left(2 n^{2}+4 n+3\right) \mu+n \rho g R\right](n-1)}\left[\frac{r(A)}{R}\right]^{n} T_{n}(A) \\
\sum \rho V_{n}(A)=\sum \rho T_{n}(A)+\sum \frac{3 n \rho^{2} R g}{2\left[\left(2 n^{2}+4 n+3\right) \mu+n \rho g R\right](n-1)}\left[\frac{r(A)}{R}\right]^{n} T_{n}(A) \\
P=\sum \frac{3 n \rho^{2} R g}{2\left[\left(2 n^{2}+4 n+3\right) \mu+n \rho g R\right](n-1)}\left[\frac{r(A)}{R}\right]^{n} T_{n}(A)+\sum \rho T_{n}(A)-\sum \frac{\left(2 n^{2}+5 n+3\right) \rho \mu}{\left(2 n^{2}+4 n+3\right) \mu+n \rho g R} T_{n}(A)
\end{gathered}
$$

When $n=0$ and $n=1, P$ is small. $T_{n}(A)$ is small when $n>2$. We set $n=2$ so that we can calculate it approximately:

$$
P=\sum \frac{3 \rho^{2} R g}{(19 \mu+2 \rho g R)}\left[\frac{r(A)}{R}\right]^{2} T_{n}(A)+\sum \rho T_{n}(A)-\sum \frac{21 \rho \mu}{19 \mu+2 \rho g R} T_{n}(A)
$$

The tidal force potential function $T_{n}(A)$ on Point A caused by the moon is

$$
T_{n}(A)=G \frac{M}{r_{m}}\left[\frac{r(A)}{r_{m}}\right]^{2} P_{2}\left(\cos Z_{m}\right)
$$

where $P_{2}\left(\cos Z_{m}\right)$ and $P_{2}\left(\cos Z_{S}\right)$ are second-order Legendre polynomials. We conclude that

$$
T_{n}(A)=G \frac{M}{2 r_{m}^{3}}\left(r^{2}(A)\right)\left(3 \cos ^{2} Z_{m}-1\right)
$$

By substituting (2) into (1), the equation for the radial stress on Point A exerted by the lunar tidal force in terms of zenith distance can be derived:

$$
P_{m}=\frac{3 \rho^{2} g}{(19 \mu+2 \rho g R) R} \frac{G M}{2 r_{m}^{3}} r^{4}(A)\left(3 \cos ^{2} Z_{m}-1\right)+\left[\rho-\frac{21 \mu \rho}{(19 \mu+2 \rho g R)}\right] \frac{G M}{2 r_{m}^{3}} r^{2}(A)\left(3 \cos ^{2} Z_{m}-1\right)
$$

Likewise, the radial stress on Point A exerted by the solar tidal force in terms of zenith distance can be described as:

$$
P S=\frac{3 \rho^{2} g}{(19 \mu+2 \rho g R) R} \frac{G S}{2 r_{S}^{3}} r^{4}(A)\left(3 \cos ^{2} Z_{S}-1\right)+\left[\rho-\frac{21 \mu \rho}{(19 \mu+2 \rho g R)}\right] \frac{G S}{2 r_{S}^{3}} r^{2}(A)\left(3 \cos ^{2} Z_{S}-1\right)
$$

The coefficients are defined as follows:

$$
C_{m 1}=\frac{3 \rho^{2} g}{(19 \mu+2 \rho g R) R} \frac{G M}{2 r_{m}^{3}} \quad C_{m 2}=\left[\rho-\frac{21 \mu \rho}{(19 \mu+2 \rho g R)}\right] \frac{G M}{2 r_{m}^{3}}
$$




$$
C_{s 1}=\frac{3 \rho^{2} g}{(19 \mu+2 \rho g R) R} \frac{G S}{2 r_{S}^{3}} \quad C_{S 2}=\left[\rho-\frac{21 \mu \rho}{(19 \mu+2 \rho g R)}\right] \frac{G S}{2 r_{S}^{3}}
$$

The upward radial stress $P_{m}$ generated by the lunar tidal force on Point A in the mantle close to the surface can be determined via

$$
P_{m}=\left[C_{m 1} r^{4}(A)+C_{m 2} r^{2}(A)\right]\left(3 \cos ^{2} Z_{m}-1\right)
$$

and the upward radial stress $P_{S}$ generated by the solar tidal force on Point A in the mantle close to the surface can be determined via

$$
P_{S}=\left[C_{s 1} r^{4}(A)+C_{s 2} r^{2}(A)\right]\left(3 \cos ^{2} Z_{s}-1\right)
$$

where

$Z_{m}$ is the zenith distance between Point A and the moon,

$Z_{S}$ is the zenith distance between Point $\mathrm{A}$ and the sun,

$r(A)$ is the distance between Point A and the earth's core,

$R$ is the average radius of the earth and for crust close to the surface, $r(A)=R$,

$\rho$ is the average density of the earth,

$G$ is the constant of universal gravitation,

$M$ is the mass of the moon,

$S$ is the mass of the sun,

$g$ is the mean acceleration of gravity,

$r_{m}$ is the distance between the centers of the moon and the earth,

$r_{S}$ is the distance between center of the solar and the earth,

$\mu$ is the average shear elasticity (rigidity modulus) of Kelvin's elastic earth model.

Let

$$
\begin{aligned}
& G=6.67259 \times 10^{-11} \mathrm{~N} \cdot \mathrm{m}^{2} / \mathrm{kg}^{2} \\
& M=7.350 \times 10^{22} \mathrm{~kg}, \\
& S=1.989 \times 10^{30} \mathrm{~kg}, \\
& r_{m}=3844 \times 10^{5} \mathrm{~m}, \\
& r_{S}=1496 \times 10^{8} \mathrm{~m} \\
& \rho=5520 \mathrm{~kg} / \mathrm{m}^{3}, \\
& g=9.82 \mathrm{~m} / \mathrm{s}^{2}, \\
& R=6371 \times 10^{3} \mathrm{~m}
\end{aligned}
$$

For the earth's surface at a geocentric distance of $r(A)=R$,

Because the thickness of the crust is trivial in comparison to the radius of the earth, the crust can be considered a thin shell, and thus the theory of non-bending moment membrane stress can be used to analyze the mechanical effect of tidal forcing on the crust. That is, under the tidal force, the upward inner pressure $P_{m}$ exerted by the viscoelastic mantle on the crust generates a horizontal extensional membrane stress within the solid crust (Figure 1B), similar to the tension of a sail caused by the wind.

According to membrane stress theory, the formulas for calculating the membrane stress on the thin shell of an axially symmetric rotating ball under inner pressure should be 


$$
\begin{gathered}
\sigma_{Z m}=\frac{1}{t R \sin ^{2} Z_{m}} \int_{0}^{Z_{m}}\left[R^{2} \sin Z_{m} \cos Z_{m} \times P_{m}\right] d Z_{m} \\
\frac{\sigma_{Z m}+\sigma_{\theta m}}{R}=\frac{P_{m}}{t}
\end{gathered}
$$

In (7) and (8),

$t$ is the thickness of the crust,

$\sigma_{Z m}$ is horizontal membrane stress in the direction of the zenith,

$\sigma_{\theta m}$ is horizontal membrane stress perpendicular to $\sigma_{Z m}$.

By substituting $P_{m}$ from (5) into (7),

$$
\sigma_{Z m}=\frac{C_{m 1} R^{5}+C_{m 2^{2}}{ }^{3}}{t \sin ^{2} Z_{m}} \int_{0}^{Z_{m}}\left[3 \sin Z_{m} \cos ^{3} Z_{m}-\sin Z_{m} \cos Z_{m}\right] d Z_{m}
$$

By integrating (9), we obtain

$$
\sigma_{Z m}=\frac{C_{m 1} R^{5}+C_{m 2} R^{3}}{4 t \sin ^{2} Z_{m}}\left(2 \cos ^{2} Z_{m}-3 \cos ^{4} Z_{m}+1\right)
$$

By substituting (10) into (8), and using $P_{m}$ from (5), we obtain

$$
\begin{gathered}
\sigma_{\theta m}=\frac{\left(C_{m 1} R^{5}+C_{m 2} R^{3}\right)\left(3 \cos ^{2} Z_{m}-1\right)}{t}-\frac{C_{m 1} R^{5}+C_{m 2} R^{3}}{4 t \sin ^{2} Z_{m}}\left(2 \cos ^{2} Z_{m}-3 \cos ^{4} Z_{m}+1\right) \\
\text { Where } C_{m 1}=\frac{3 \rho^{2} g}{(19 \mu+2 \rho g R) R} \frac{G M}{2 r_{m}^{3}} \quad C_{m 2}=\left[\rho-\frac{21 \mu \rho}{(19 \mu+2 \rho g R)}\right] \frac{G M}{2 r_{m}^{3}}
\end{gathered}
$$

Equations (10) and (11) are the final formulas for the extensional membrane stress on Point $\mathrm{A}$ in the crust caused by the lunar tidal forcing of the viscoelastic mantle, where $\sigma_{Z m}$ is the horizontal membrane stress in the direction of the lunar zenith and $\sigma_{\theta m}$ is the horizontal membrane stress perpendicular to $\sigma_{Z m}$.

Similarly, the extensional membrane stress on Point A in the crust caused by the viscoelastic mantle driven by solar tidal forcing can be calculated via the following formulas:

$$
\begin{gathered}
\sigma_{Z s}=\frac{C_{S 1} R^{5}+C_{s 2} R^{3}}{4 t \sin ^{2} Z_{S}}\left(2 \cos ^{2} Z_{S}-3 \cos ^{4} Z_{S}+1\right) \\
\sigma_{\theta s}=\frac{\left(C_{s 1} R^{5}+C_{s 2} R^{3}\right)\left(3 \cos ^{2} Z_{S}-1\right)}{t}-\frac{C_{s 1} R^{5}+C_{s 2} R^{3}}{4 t \sin ^{2} Z_{S}}\left(2 \cos ^{2} Z_{S}-3 \cos ^{4} Z_{S}+1\right)
\end{gathered}
$$

$$
\text { Where } C_{S 1}=\frac{3 \rho^{2} g}{(19 \mu+2 \rho g R) R} \frac{G S}{2 r_{S}^{3}}, \quad C_{S 2}=\left[\rho-\frac{21 \mu \rho}{(19 \mu+2 \rho g R)}\right] \frac{G S}{2 r_{S}^{3}}
$$

In formulas (13) and (14),

$\sigma_{Z s}$ is the horizontal membrane stress in the direction of the solar zenith, 
$\sigma_{\theta s}$ is the horizontal membrane stress in the direction of $\sigma_{Z s}$.

\section{Results}

Fig. 2 shows an earth model based on PREM. The magnitude of the shear modulus $\mu$ is hypothesized to vary from 1.0E10 to 5.0E11, and the crust membrane stress can be obtained from the above equations. From Figure 2, we can see that when the crustal thickness is less than $10 \mathrm{~km}$, a seemingly insignificant tidal force would cause a horizontal tensile membrane stress within the crust, enabling tension fracturing (the tensile fracture strength for rocks is approximately $4-6 \mathrm{MPa}$ ).
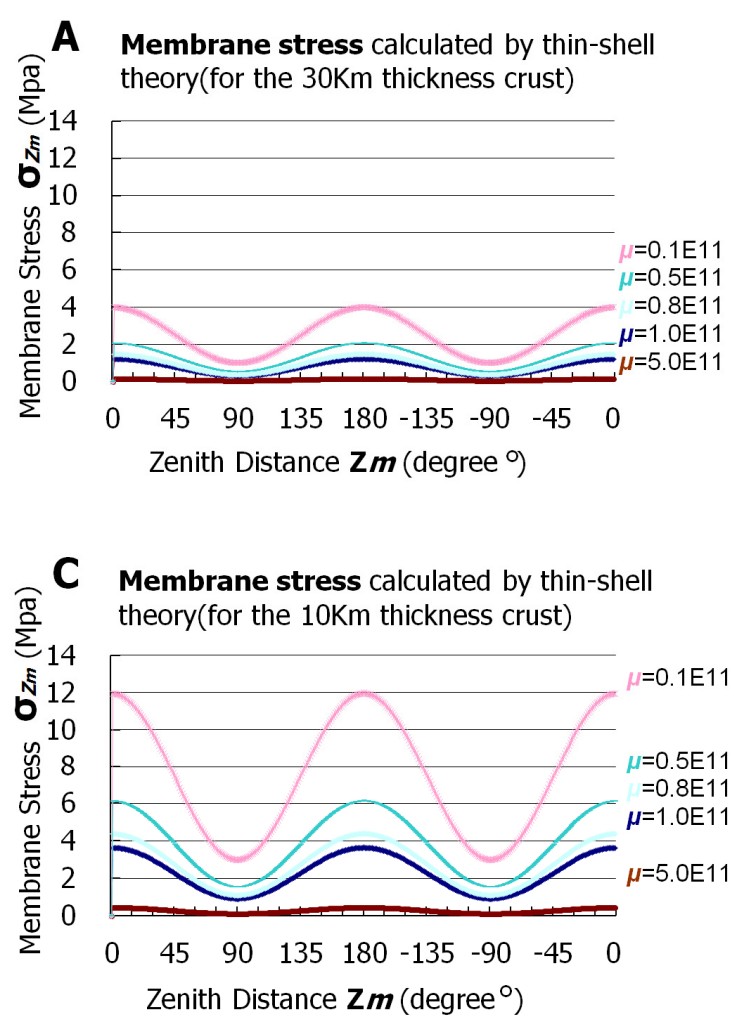
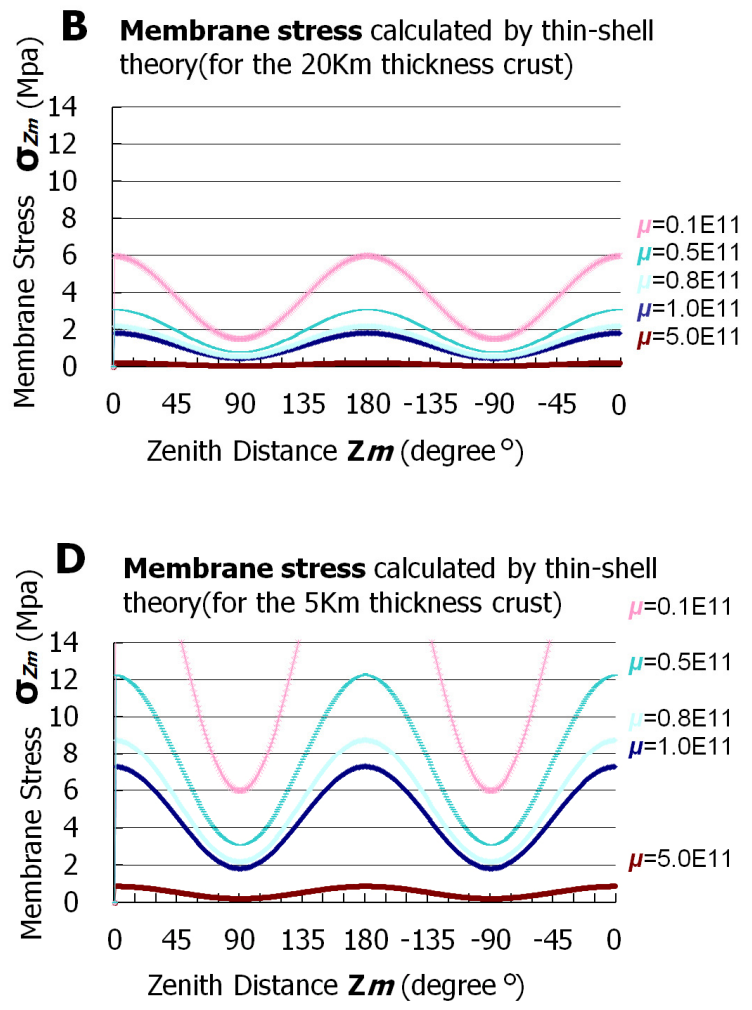

Figure 2. The membrane stresses on crust of various thicknesses are calculated using the thin-shell theory. Although the tide-generating force is seemingly insignificant, the horizontal tensile membrane stress caused by the solid tide in crust less than $10 \mathrm{~km}$ thick is powerful enough to cause tension fractures in rocks with strengths up to $5 \mathrm{MPa}$.

\section{Discussion}

We believe that fracturing of crust less than $10 \mathrm{~km}$ thick will be intensified and spread farther with the continued cyclic deformation caused by the tide-generating force. At mid-ocean ridges, lava is continuously erupted. Although the lava solidifies when it meets sea water, the broken crust does not have enough time to solidify completely due to the tensile effects of the semidiurnal solid tide. In other words, the crust has been ruptured since the existence of the moon and will continue to be in the future.

Therefore, we believe that both the rupturing of the crust and the formation of mid-ocean ridges are caused by the tensile membrane stress due to the tidal force (Figure 3A). In addition, under the periodic vibration of the solid tide, lava at mid-ocean ridges accumulates gradually and experiences repeated cycles of eruption and cooling. When a sufficient amount of solidified lava has accumulated, gravity causes the lava to thrust horizontally over the mid-ocean ridge and the oceanic plates on both sides of the ridge via wedging, giving rise to pressured seafloor spreading and plate motion due to the solid tide. This cycle of eruption and cooling, followed by wedging has a stable frequency in response to the cycle of earth tides, and thus the plate motion has become stable over time. Figure 3B depicts the breaking of a mid-ocean ridge and the consequent surging of 
mantle materials caused by the tide-generating force, as well as the resulting plate motion and seafloor spreading. Due to the randomness of the horizontal component of the membrane stress, triple junctions may form. Transform fault perpendicular to mid-ocean ridges also form through this process.
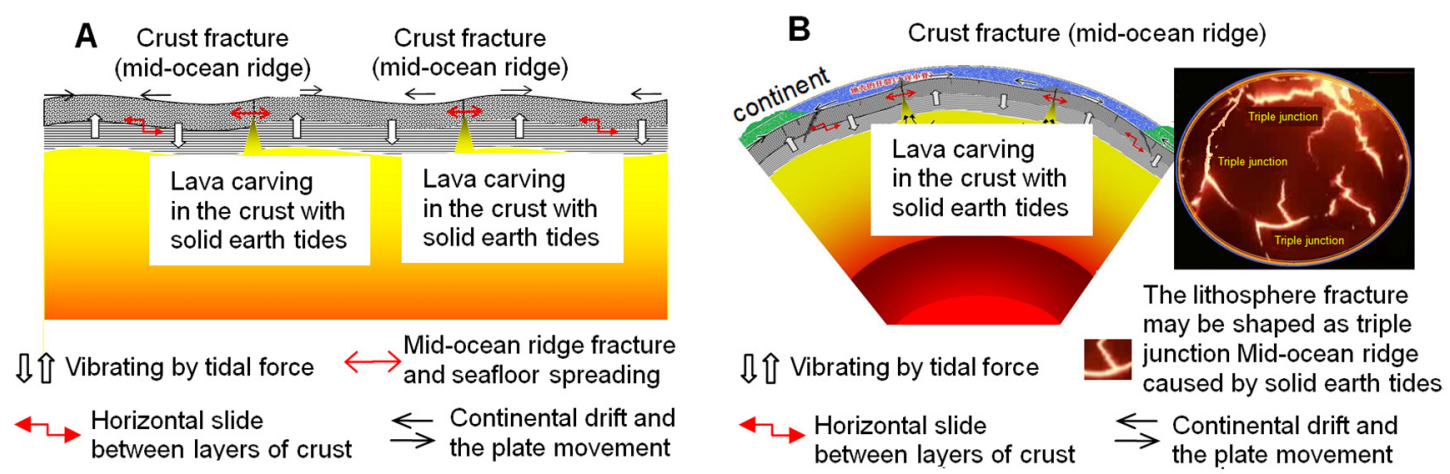

Figure 3. (A) While triggering numerous earthquakes, the tide-generating force can also result in fracturing at mid-ocean ridges and a surge in lava from the mantle. This causes plates to move and leads to seafloor spreading, causing plate motion. (B) The membrane stress in the crust is caused by the earth tides and is tensile in any horizontal direction. Due to membrane stress, transformation faults are perpendicular to the mid-ocean ridges and large fault zones or ridges may form triple junctions, which are not explained by mantle convection or mantle plume models

Therefore, we propose that the thin-shell theory can be used to analyze the dynamic effect of the tidal force on the crust. Our findings are not only a theoretical support of the proposition that mid-ocean ridge fracturing and plate motions originate from the lunar-solar tidal force, but they are also a possible solution to certain geological problems, including the long-term stabilization of plate motions, the formation of triple junctions, large transform faults and why only the earth has tectonic plates. These explanations make the present theory more rational than mantle convection models and other previous geodynamical theories.

Although other terrestrial planets in our solar system have concentric layers such as the core, mantle and crust, and they may have mantle convection similar to the earth, they do not have seafloor spreading or tectonic plates.

We believe that the earth has three specific conditions that make it possible for it to have tectonic plates and the capacity for life, arising from the existence of the moon. These three conditions are as follows:

1) Among all the terrestrial planets, the moon has a relatively large mass relative to the earth and is relatively close to the earth, which causes a strong tide-generating force.

2) Affected by the tidal force, the earth has a viscoelastic mantle capable of exerting a radial stress and a membrane stress on the solid crust.

3) The thickness of the earth's crust relative to its radius is very small and the membrane stress is strong enough to cause crust thinner than $10 \mathrm{~km}$ to rupture, leading to large-scale mid-ocean ridges and tectonic plates.

For the first time, the thin-shell mechanism is used to analyze the response of the crust under the tide-generating force and to speculate about the driving source of plate motions. And we may discover the plate tectonics effected by the tidal force is in the terrestrial planets or other celestial bodies in future.

The conclusions of our analysis are:

1) Although the tidal force is small, the membrane stress is large. A theoretical formula is given for the crust membrane stress model. Calculations show that the horizontal tensile membrane stress is capable of causing tensile rupture in oceanic crust.

2) We speculate that the tide-generating force drives plate motions. We believe that the solid tide triggers numerous earthquakes, and the membrane stress within the crust makes possible for triple junctions and large transform faults to form in the thinner parts of the oceanic crust. Seafloor spreading and plate motions arise from the membrane stress and are formed by lava that has erupted due to the tide-generating force. The existence of the moon is the reason why the earth become the only planet in our solar system to have tectonic plates and plate 
motions. The analysis of shell mechanics may cause new breakthroughs in geodynamics.

3) We infer that there must be the plate tectonics effected by the tidal force is in the terrestrial planets or other celestial bodies.

\section{Acknowledgements}

This research was supported by the Key National Science Foundation of China (Grant No. 40730103), the National Science Foundation of China (Grant No. 40571166, 40672107), and the National Science and Technology Support Program (Grant No. 2006BAC01B02-02-05)

\section{References}

Belytschko, T., Stolarski, H., Liu, W. K., Carpenter, N., \& Ong, J. S. J. (1985). Stress projection for membrane and shear locking in shell finite-elements. Computer Methods In Applied Mechanics and Engineering, 51(1-3), 221-258. http://dx.doi.org/10.1016/0045-7825(85)90035-0

Bird, P. (1998). Testing hypotheses on plate-driving mechanisms with global lithosphere models including topography, thermal structure, and faults. Journal of Geophysical Research-Solid Earth, 103(B5), 10115-10129. http://dx.doi.org/10.1029/98JB00198

Bitbol, A. F., Peliti, L., \& Fournier, J. B. (2011). Membrane stress tensor in the presence of lipid density and composition inhomogeneities. European Physical Journal E, 34(5). http://dx.doi.org/10.1140/epje/i2011-11053-4

Capovilla, R., \& Guven, J. (2002). Stresses in lipid membranes. Journal Of Physics A-mathematical and General, 35(30), 6233-6247. http://dx.doi.org/10.1088/0305-4470/35/30/302

Chen, L., Chen, J. G., \& Xu, Q. H. (2012). Correlations between solid tides and worldwide earthquakes ms \&ge; 7.0 since 1900. Nat. Hazards Earth Syst. Sci., 12(3), 587-590. http://dx.doi.org/10.5194/nhess-12-587-2012

Conrad, C. P., \& Lithgow-Bertelloni, C. (2004). The temporal evolution of plate driving forces: Importance of "slab suction" versus "slab pull" during the cenozoic. Journal of Geophysical Research-Solid Earth, 109(B10).

Evans, J. W. (1923). The wegener hypothesis of continental drift. Nature, 111, 393-394. http://dx.doi.org/10.1038/111393a0

Forsyth, D., \& Uyeda, S. (1975). Relative importance of driving forces of plate motion. [Review]. Geophysical $\begin{array}{llll}\text { Journal of the Royal Astronomical Society, } & \text { 43(1), }\end{array}$ http://dx.doi.org/10.1111/j.1365-246X.1975.tb00631.x

Hallam, A. (1975). Wegener,a and hypothesis of continental-drift. Scientific American, 232(2), 88-\&. http://dx.doi.org/10.1038/scientificamerican0275-88

Jordan, T. H. (1974). Some comments on tidal drag as a mechanism for driving plate motions. Journal Of Geophysical Research, 79(14), 2141-2142. http://dx.doi.org/10.1029/JB079i014p02141

Lithgowbertelloni, C., \& Richards, M. A. (1995). Cenozoic plate driving forces. Geophysical Research Letters, 22(11), 1317-1320. http://dx.doi.org/10.1029/95GL01325

Meinesz, F. A. V. (1947). Shear patterns of the earth's crust. Transactions of the American Geophysical Union, 28, $1-61$.

Minster, J. B., Jordan, T. H., Molnar, P., \& Haines, E. (1974). Numerical modeling of instantaneous plate tectonics. Geophysical Journal of the Royal Astronomical Society, 36(3), 541-576. http://dx.doi.org/10.1111/j.1365-246X.1974.tb00613.x

Mobus, G. (1982). In memory of wegener,alfred - on the occasion of the 70th anniversary of his 1st publication on his continental-drift hypothesis. Petermanns Geographische Mitteilungen, 126(3), 137-148.

Person, M., \& Garven, G. (1994). A sensitivity study of the driving forces on fluid-flow during continental-drift basin evolution. Geological Society Of America Bulletin, 106(4), 461-475. http://dx.doi.org/10.1130/0016-7606(1994)106<0461:ASSOTD>2.3.CO;2

Plattner, C., Malservisi, R., \& Govers, R. (2009). On the plate boundary forces that drive and resist baja california motion. Geology, 37(4), 359-362. http://dx.doi.org/10.1130/G25360A.1

Ranalli, G. (2000). Westward drift of the lithosphere: Not a result of rotational drag. Geophysical Journal International, 141(2), 535-537. http://dx.doi.org/10.1046/j.1365-246x.2000.00091.x 
Royden, L. H. (1993). The tectonic expression slab pull at continental convergent boundaries. Tectonics, 12(2), 303-325. http://dx.doi.org/10.1029/92TC02248

Scoppola, B., Boccaletti, D., Bevis, A., Carminati, E., \& Doglioni, C. (2006). The westward drift of the lithosphere: A rotational drag? (vol 118, pg 199, 2006). Geological Society Of America Bulletin, 118(3-4), 511-511.

Springer, F., Ghidossi, R., Carretier, E., Veyret, D., Dhaler, D., \& Moulin, P. (2010). Study of the effect of geometry on wall shear stress and permeate flux for ceramic membranes: Cfd and experimental approaches. Engineering Applications of Computational Fluid Mechanics, 4(1), 17-28.

Vandecar, J. C., James, D. E., \& Assumpcao, M. (1995). Seismic evidence for a fossil mantle plume beneath south-america and implications for plate driving forces. Nature, 378(6552), $25-31$. http://dx.doi.org/10.1038/378025a0

Wahr, J., Selvans, Z. A., Mullen, M. E., Barr, A. C., Collins, G. C., Selvans, M. M., \& Pappalardo, R. T. (2009). Modeling stresses on satellites due to nonsynchronous rotation and orbital eccentricity using gravitational potential theory. Icarus, 200(1), 188-206. http://dx.doi.org/10.1016/j.icarus.2008.11.002 